\title{
The Usefulness of Surgical Treatment in Slow-Flow Vascular Malformation Patients
}

\author{
Gyu Bin Kang ${ }^{1}$, Yong Chan Bae ${ }^{1,2}$, Su Bong Nam¹, Seong Hwan Bae ${ }^{1}$, Ji Yoon Sung ${ }^{1}$ \\ ${ }^{1}$ Department of Plastic and Reconstructive Surgery, Pusan National University School of Medicine, Busan; ${ }^{2}$ Biomedical Research Institute, \\ Pusan National University Hospital, Busan, Korea
}

Background Many difficulties exist in establishing a treatment plan for slow-flow vascular malformation (SFVM). In particular, little research has been conducted on the surgical treatment of SFVMs. Thus, we investigated what proportion of SFVM patients were candidates for surgical treatment in clinical practice and how useful surgical treatment was in those patients.

Methods This study included 109 SFVM patients who received care at the authors' clinic from 2007 to 2015. We classified the patients as operable or non-operable, and analyzed whether the operability and the extent of the excision varied according to the subtype and location of the SFVM. Additionally, we investigated complications and self-assessed satisfaction scores.

Results Of the 109 SFVM patients, 59 (54\%) were operable, while $50(46 \%)$ were nonoperable. Total excision could be performed in 44\% of the operable SFVM patients. Lymphatic malformations were frequently non-operable, while capillary malformations were relatively operable $(P=0.042)$. Total excision of venous malformations could generally be performed, while lymphatic malformations and combined vascular malformations generally could only undergo partial excision $(\mathrm{P}=0.048)$. Complications occurred in $11 \%$ of the SFVM patients who underwent surgery; these were minor complications, except for 1 case. The average overall satisfaction score was 4.19 out of 5 .

Conclusions Based on many years of experience, we found that approximately half (54\%) of SFVM patients were able to undergo surgery, and around half (44\%) of those patients were able to fully recover after a total excision.

Among the patients who underwent surgical treatment, high satisfaction was found overall and relatively few complications were reported.

Keywords Vascular malformations / Treatment outcome / Follow-up studies
Correspondence: Yong Chan Bae Department of Plastic and

Reconstructive Surgery, Biomedical Research Institute, Pusan National University Hospital, Pusan National University School of Medicine, 179 Gudeok-ro, Seo-gu, Busan 49241, Korea

Tel: +82-51-240-7273

Fax: +82-51-243-9405

E-mail: baeyc2@hanmail.net

Received: 29 Oct 2016 • Revised: 13 Feb 2017 • Accepted: 28 Mar 2017

pISSN: 2234-6163 • elSSN: 2234-6171 • https://doi.org/10.5999/aps.2017.44.4.301 • Arch Plast Surg 2017;44:301-307

This work was supported by a clinical research grant from Pusan National University Hospital in 2017.

No potential conflict of interest relevant to this article was reported.

\section{INTRODUCTION}

According to the International Society for the Study of Vascular Anomalies, vascular malformations are divided into slow-flow and fast-flow vascular malformations. In addition, slow-flow vascular malformations (SFVMs) are divided into capillary, venous, lymphatic, and combined vascular malformations [1-3]. Many difficulties exist in establishing a treatment plan for 
SFVMs due to the difficulty of diagnosing them accurately and the variety of treatment methods. Recently, increasing interest has emerged in minimally invasive treatment, and McCafferty [4] and Lee [5] consider non-surgical treatment such as sclerotherapy to be an important option for the treatment of vascular malformations.

Although some studies have reported that surgical treatment was associated with a high recurrence rate as well as a high complication rate, systematic, long-term, and large-scale research has not been conducted on the surgical treatment of SFVM. In particular, little research has been conducted to determine what proportion of SFVM patients can receive surgical treatment in clinical practice and how useful surgical treatment is for such patients.

Thus, the authors analyzed the SFVM patients who received treatment at the Vascular Malformation Clinic, which is the authors' multidisciplinary clinic, based on whether surgical treatment could be performed and how much of the vascular malformation could be excised. In addition, we examined whether the possibility of surgical treatment varied according to the subtype and location of the lesion and investigated postoperative satisfaction and complications in the SFVM patients who underwent surgery.

\section{METHODS}

\section{Subjects}

In this study, we analyzed the medical records, interviews, and telephone surveys of 109 SFVM patients who underwent adequate follow-up among the patients who visited the Vascular Malformation Clinic from 2007 to 2015. Our sample included 55 males and 54 females, an approximately 1:1 ratio, and no significant difference in incidence was observed according to sex. The cases were divided by subtype into 21 capillary malformations, 11 lymphatic malformations, 71 venous malformations, 4 capillary-venous malformations, and 2 capillary-lymphatic-ve-

\begin{tabular}{|c|c|c|c|}
\hline Subtype & Male & Female & Subtotal \\
\hline CM & 12 & 9 & 21 \\
\hline LM & 5 & 6 & 11 \\
\hline VM & 36 & 35 & 71 \\
\hline Combined VM & 2 & 4 & 6 \\
\hline Total & 55 & 54 & 109 \\
\hline \multicolumn{4}{|c|}{$\begin{array}{l}\text { Values are presented as numbers. } \\
\text { SFVM, slow-flow vascular malformation; CM, capillary malformation; LM } \\
\text { lymphatic malformation; VM, venous malformation; combined VM, combine } \\
\text { vascular malformation. }\end{array}$} \\
\hline
\end{tabular}

nous malformations (Table 1 ).

The patients ranged in age from 3 months to 69 years, and their mean age was $21.8 \pm 16.5$ years at the time of treatment.

SFVMs occur most frequently in the head and neck, followed by the upper extremities, lower extremities, and trunk, in order. Multiple SFVMs occurred in $8 \%$ of the patients ( 9 cases) (Table 2 ).

\section{Methods}

The SFVM patients were divided according to whether surgical treatment could be performed, and postoperative complications and satisfaction were evaluated for the SFVM patients who underwent surgery through a review of their medical records and a survey.

\section{Possibility of surgical treatment}

In this study, the SFVM patients were divided into operable patients and non-operable patients, and then the operable patients were divided based on whether a total or partial excision could be performed.

In addition, a statistical analysis was performed to determine whether operability and the extent of the excision varied according to the subtype and location of the lesion.

\section{Satisfaction and complications}

The postoperative satisfaction of the SFVM patients was assessed using a questionnaire. Of the 59 patients who were encouraged to undergo surgery, 45 ultimately underwent surgical treatment, and 32 of those patients responded to the survey (response rate, $71 \%$ ). The patients rated their overall satisfaction regarding their overall appearance, symptoms, and quality of

\begin{tabular}{|c|c|c|c|c|}
\hline \multirow[b]{2}{*}{ Subtype } & \multicolumn{2}{|c|}{ Operable } & \multirow{2}{*}{$\begin{array}{c}\text { Non- } \\
\text { operable }\end{array}$} & \multirow[b]{2}{*}{ Subtotal } \\
\hline & $\begin{array}{c}\text { Total } \\
\text { excision }\end{array}$ & $\begin{array}{c}\text { Partial } \\
\text { excision }\end{array}$ & & \\
\hline \multirow[t]{2}{*}{ CM } & \multicolumn{2}{|c|}{$16(76)$} & $5(24)$ & $21(100)$ \\
\hline & $6(28)$ & $10(48)$ & & \\
\hline \multirow[t]{2}{*}{ LM } & \multicolumn{2}{|c|}{$3(27)$} & $8(73)$ & $11(100)$ \\
\hline & 0 & $3(27)$ & & \\
\hline \multirow[t]{2}{*}{ VM } & \multicolumn{2}{|c|}{$36(50)$} & $35(50)$ & $71(100)$ \\
\hline & $20(28)$ & $16(22)$ & & \\
\hline \multirow[t]{2}{*}{ Combined VM } & \multicolumn{2}{|c|}{$4(66)$} & $2(34)$ & $6(100)$ \\
\hline & 0 & $4(66)$ & & \\
\hline \multirow[t]{2}{*}{ Total } & \multicolumn{2}{|c|}{$59(54)$} & $50(46)$ & 109 (100) \\
\hline & $26(24)$ & $33(30)$ & & \\
\hline
\end{tabular}


life. They were asked to evaluate the results of surgery and their satisfaction using a 5-point Likert scale, in which scores of 1, 2, 3, 4 , and 5 indicated poor, fair, average, good, and excellent levels of satisfaction, respectively. The survey was performed at least 1 year after treatment. The average postoperative follow-up period was 56 months, ranging from 12 months to 11 years.

Additionally, all postoperative complications were evaluated based on the patients' medical records.

The chi-square test and the Fisher exact test were used to evaluate operability and the extent of the excision according to subtype and lesion location, while differences in satisfaction according to subtype were analyzed using the Kruskal-Wallis test. P-values $<0.05$ were considered to indicate statistical significance. All statistical results were obtained using SPSS ver. 22.0 (IBM, Armonk, NY, USA).

\section{RESULTS}

\section{Operability}

This study divided SFVM patients based on operability because many of the 109 SFVM patients received more than one type of treatment. It was determined that 59 of the patients (54\%) patients were operable, while $50(46 \%)$ patients were non-operable (Table 2).

Venous malformations were divided into localized and diffuse venous malformations. Of the 34 localized venous malformations, 22 were operable, whereas only 14 of the 37 diffuse venous malformations were operable.

Lymphatic malformations tended to be non-operable, whereas capillary malformations were relatively operable.

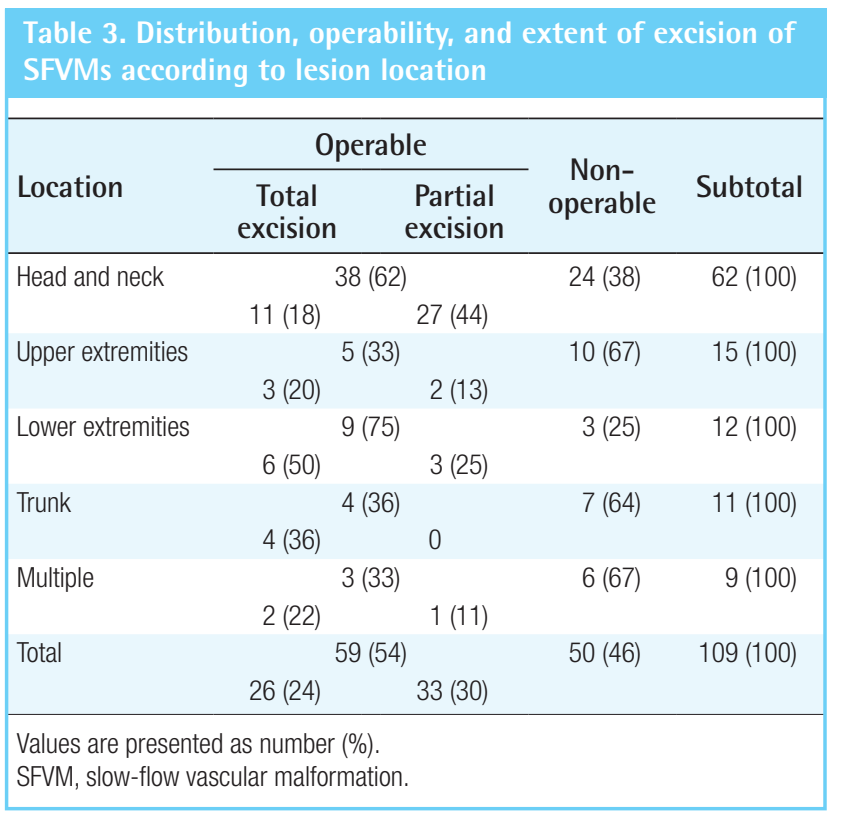

Operable patients were then subdivided according to the extent of excision. It was possible to perform total excision in 26 of the operable SFVM patients (44\%), while partial excision was possible to performed in the remaining 33 (56\%) (Table 2).

In addition, many patients with venous malformations could undergo total excision, whereas patients with lymphatic malformations and combined vascular malformations generally could only undergo partial excision.

Operability $(\mathrm{P}=0.042)$ and the extent of the excision $(\mathrm{P}=$ 0.048 ) varied significantly according to subtype.

It was often difficult for patients with SFVMs in their upper extremities, trunk, or multiple locations to undergo surgery, whereas was more likely to be possible for patients with SFVMs in their lower extremities. However, this trend was not statistically significant $(\mathrm{P}=0.064)$ (Table 3$)$.

Moreover, many patients with an SFVM in their trunk could undergo total excision, whereas relatively few patients with an SFVM in their head and neck region could undergo total excision. This trend was statistically significant $(\mathrm{P}=0.009)$ (Table 3 ).

The 50 non-operable patients were recommended to undergo sclerotherapy, laser treatment, or observation. Laser treatment was recommended to 5 patients with a capillary malformation. Sclerotherapy was recommended to 33 patients with a venous malformation, 7 patients with a lymphatic malformation, and 1 patient with a combined vascular malformation. Observation was recommended to 4 patients (Table 4).

\section{Satisfaction and complications}

In the satisfaction survey of the patients who underwent surgical treatment, the score for patients who underwent total excision was 4.14 points, while the score for patients who underwent partial excision was 4.22 points. The mean satisfaction was $4.19 \pm 0.93$ points (range, $2-5$ points); this level reflects good to excellent satisfaction, indicating that the patients were generally

Table 4. Non-operative treatment of non-operable SFVM patients

\begin{tabular}{lcccc}
\hline \multirow{2}{*}{ Subtype } & \multicolumn{3}{c}{ Non-operative treatment } & Subtotal \\
\cline { 2 - 4 } & $\begin{array}{c}\text { Laser } \\
\text { treatment }\end{array}$ & Sclerotherapy & Observation & \\
\hline CM & 5 & - & - & 5 \\
LM & - & 7 & 1 & 8 \\
VM & - & 33 & 2 & 35 \\
Combined VM & - & 1 & 1 & 2 \\
Total & 5 & 41 & 4 & 50 \\
\hline Values are presented as number. & & \\
SFVM, slow-flow vascular malformation; CM, capillary malformation; LM, \\
lymphatic malformation; VM, venous malformation; combined VM, combined \\
vascular malformation.
\end{tabular}


satisfied with the results of surgery.

Patients with capillary, lymphatic, venous, and combined vascular malformations reported mean satisfaction scores of 4.12, $4.30,4.26$, and 3.50, respectively. These scores were analyzed using the Kruskal-Wallis test, and no statistically significant differences were found according to subtype $(\mathrm{P}=0.643)$ (Table 5).

Complications occurred in a total of 5 of the 45 SFVM patients who underwent surgical treatment (11\%). Wound dehiscence took place in 1 combined vascular malformation patient, while minor wound healing problems were noted in 2 capillary malformation patients and 2 venous malformation patients.

\section{Case A}

Case A was a 5-year-old girl with a chief complaint of a bulging deformity in the abdomen from the time of birth. She visited the Vascular Malformation Clinic and was diagnosed with a venous malformation on preoperative magnetic resonance imaging. A venous malformation was observed in the subcutaneous fat layer of the skin of the lower abdominal wall and was opera-

\begin{tabular}{|c|c|c|c|}
\hline \multirow{2}{*}{ Subtype } & \multicolumn{2}{|c|}{ Satisfaction } & \multirow{2}{*}{$\begin{array}{c}\text { Mean } \\
\text { satisfaction }\end{array}$} \\
\hline & Total excision & Partial excision & \\
\hline $\mathrm{CM}$ & 3.75 & 4.50 & 4.12 \\
\hline LM & - & 4.30 & 4.30 \\
\hline VM & 4.30 & 4.22 & 4.26 \\
\hline Combined VM & - & 3.50 & 3.50 \\
\hline Average & 4.14 & 4.22 & 4.19 \\
\hline
\end{tabular}

ble. Thus, the patient underwent total excision. She received outpatient follow-up and did not show any abnormal findings, such as recurrence or complications, at an 18-month follow-up (Fig. 1).

\section{Case B}

Case B was a 61-year-old man who visited the Vascular Malformation Clinic because his right visual field was covered by a deformity of the right upper eyelid and forehead. He was diagnosed with a capillary malformation and underwent partial excision twice. His right visual field was greatly improved after surgery, and he was followed up without complications for 30 months after surgery (Fig. 2).

\section{Case C}

Case $\mathrm{C}$ was a 40-year-old woman with a left hemifacial portwine nevus from the time of birth who was diagnosed with Sturge-Weber syndrome. She visited the Vascular Malformation Clinic, and multiple capillary-venous malformations were observed in the submandibular, sublingual, and parotid spaces, the retropharynx, the hypopharynx, and the oropharynx. Partial excision was performed on the lower lip, and liposuction was performed on the neck. After surgery we performed laser therapy and sclerotherapy, additionally. She did not display any aggravation of her symptoms or complications for 4 years after surgery (Fig. 3).

\section{Case D}

Case $\mathrm{D}$ was a 17-year-old male who was diagnosed with a capillary-venous-lymphatic malformation in the upper extremity and trunk. In magnetic resonance imaging, a capillary-venous-lym-

\section{Fig. 1. Venous malformation in the abdomen}

A 5-year-old girl who underwent total excision of a venous malformation in the abdomen. (A) Preoperative image. (B) A preoperative magnetic resonance image of low abdominal wall shows a venous malformation (red arrow). (C) A postoperative image taken 14 months after total excision surgery.
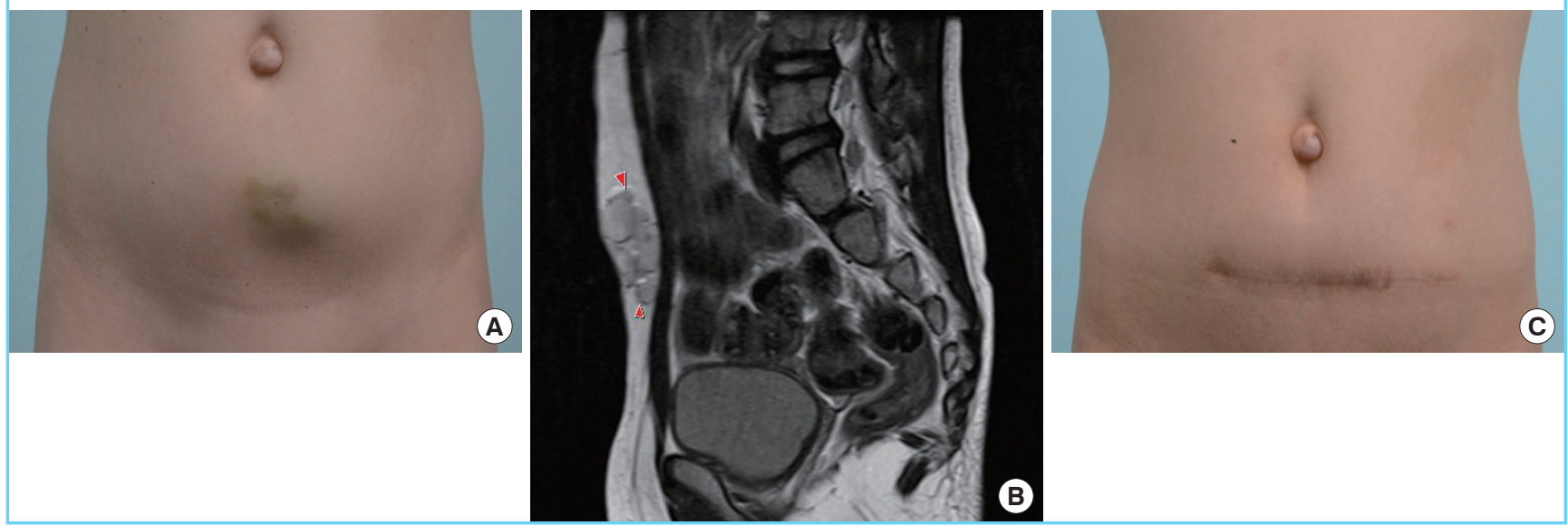


\section{Fig. 2. CM of the upper eyelid and forehead}

A 61-year-old man who underwent partial excision of a capillary malformation of the right upper eyelid and forehead. (A) Preoperative photograph. (B) A photograph after the first partial excision procedure. (C) A postoperative image taken 18 months after the second partial excision procedure.
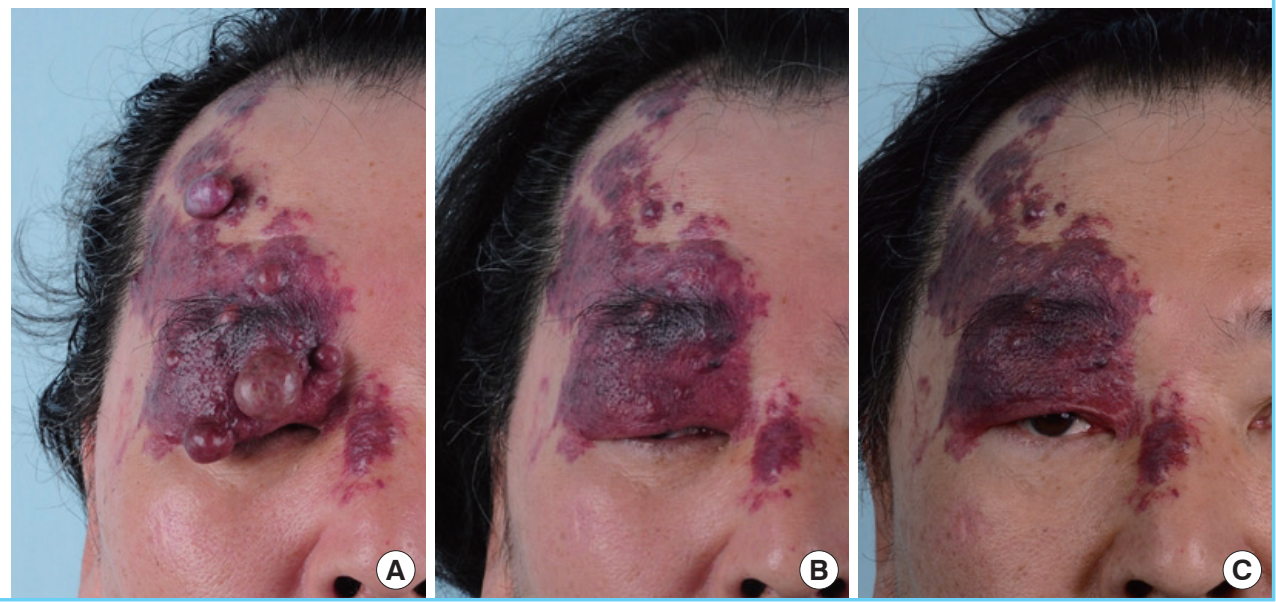

\section{Fig. 3. Combined VM on the lower lip}

A 40-year-old woman who underwent partial excision of a capillary-venous malformation on the lower lip. (A) Preoperative photograph. (B) Magnetic resonance imaging before surgery. (C) Photograph taken 3 months after surgery.
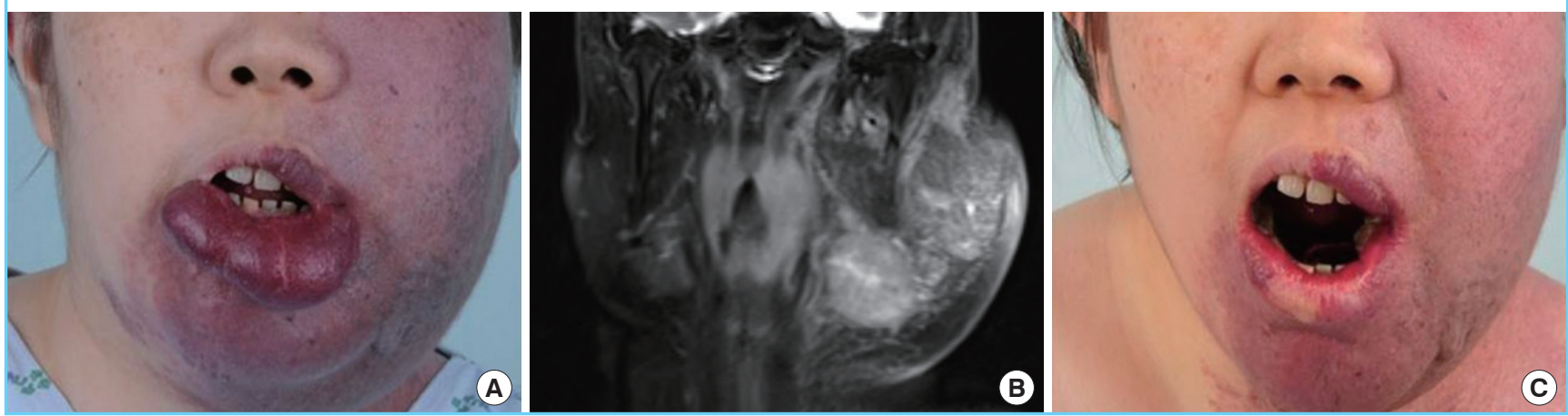

Fig. 4. Combined VM in the right arm and trunk

A 17-year-old male who underwent follow-up without surgical treatment because surgical treatment was not feasible. (A) Left upper extremity. (B) Right upper extremity. (C) A axial magnetic resonance image shows an capillary-venous-lymphatic malformation (red arrows). (D) A coronal magnetic resonance image shows an capillary-venous-lymphatic malformation (red arrows).
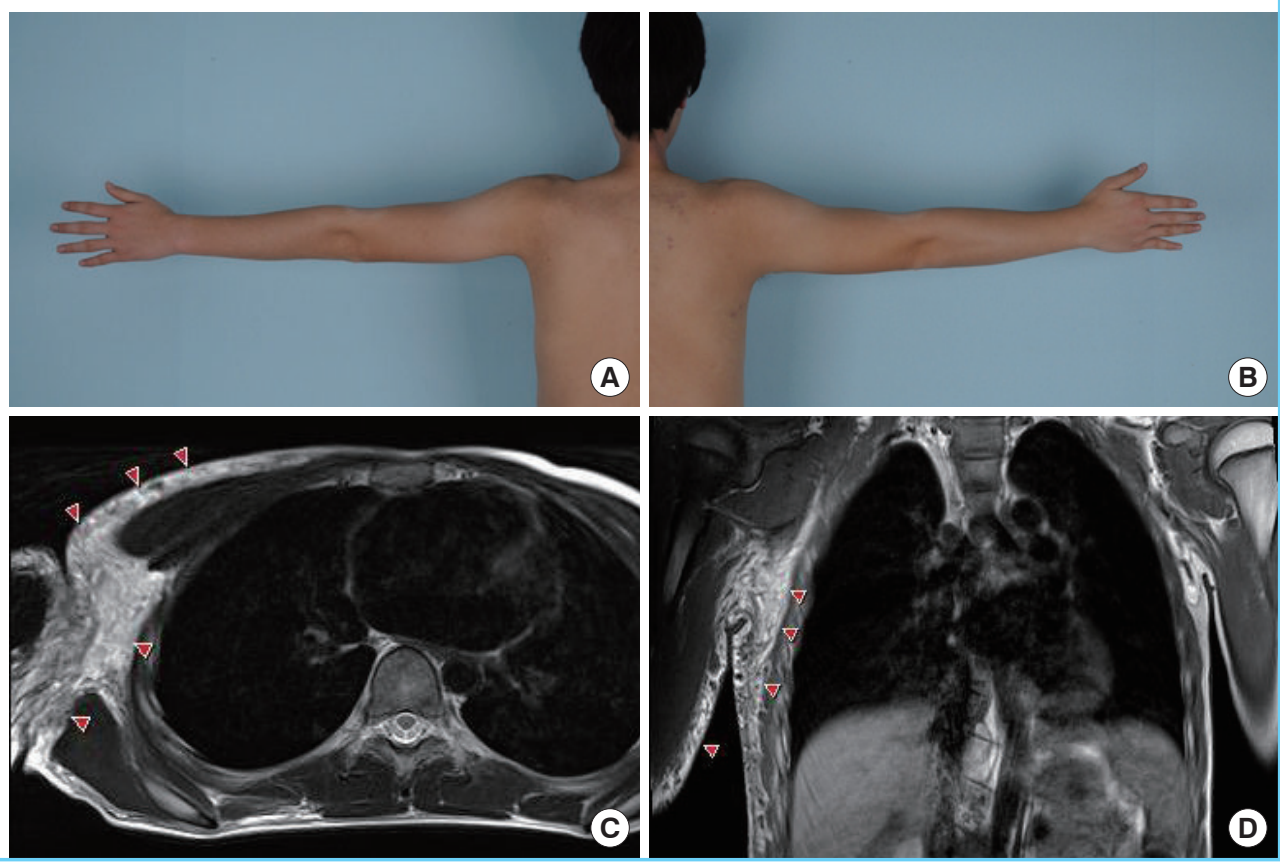
phatic malformation was observed in the right anterior, lateral, and posterior chest wall and right axilla, as well as in the right upper extremity and right supraclavicular area. The lesions were mainly located in the subcutaneous layer, but extended into the intermuscular space in the right chest wall. He underwent follow-up without surgical treatment because surgical treatment was not feasible (Fig. 4).

\section{DISCUSSION}

SFVMs are easily misdiagnosed and are often inappropriately treated. Very et al. [6] reported that a collaborative approach to the treatment of SFVMs yielded superior results. The authors of the present study opened the Vascular Malformation Clinic in 2002, and this clinic has subsequently provided collaborative treatment, with a team of dermatologists, radiologists, and plastic surgeons. Thus, medical specialists in various fields have endeavored to select the best treatment methods among options such as surgery, sclerotherapy, laser therapy, and embolism therapy.

A variety of treatment methods for SFVM have been tried because a single clear treatment method has yet to be established. Some surgeons consider non-surgical treatment such as sclerotherapy to be the preferable treatment method for SFVM. Based on previous studies, the rate of complications such as airway obstruction, scarring, hematoma, and infection may be as high as $33 \%$, and the recurrence rate may range from $15 \%$ to $53 \%$ [4].

However, Roh et al. [7] reported that surgical treatment could improve $75 \%$ of symptoms in cases where non-surgical treatments, such as sclerotherapy, failed. Steiner et al. [8] argued that surgery may still be an important treatment method because it has the advantage of allowing complete excision of the lesion and results in high patient satisfaction

In this study, we analyzed how applicable and useful surgical treatment was for SFVM patients. No standardized surgical indications for the treatment of vascular anomalies yet exist. We considered operative procedures to be reasonably feasible if surgical treatment was likely to lead to improvements in the patient's appearance, function, and quality of life, and if the patient agreed. If the lesion was well-localized or otherwise amenable to excision, we considered total excision.

Approximately half (54\%) of the SFVM patients could undergo surgery, and approximately half (44\%) of those patients were able to fully recover through total excision. High overall satisfaction was found among the patients who underwent surgical treatment, and, remarkably, the patients who underwent partial excision showed similar levels of satisfaction to those who underwent total excision.

Complications occurred in $11 \%$ of the 45 SFVM patients who underwent surgical treatment. The complications were minor, except for 1 case that needed to be revised due to wound dehiscence. The complication rate was lower than has been reported in previous studies [4].

Lymphatic malformations tended to be non-operable, and when operable, were unlikely to be completely excised. In contrast, capillary malformations were relatively operable, and many patients with venous malformations were able to undergo total excision. In particular, localized venous malformation patients were more likely to be operable than diffuse venous malformation patients.

Although many patients with SFVMs in the head and neck region could undergo surgery, total excision was difficult to perform.

Depending on the type of the SFVM and the location of the lesion, the 50 non-operable patients were recommended to undergo sclerotherapy, laser treatment, or observation.

Radical excision of SFVMs is rare. Therefore, treatment should be focused on patient satisfaction and the improvement of symptoms. It is important to consider a range of methods appropriate for patients because treatment methods are quite diverse and it is difficult to obtain satisfactory results using only a single method. Based on the authors' many years of experience, surgical treatment has been confirmed to enable some patients to fully recover through total excision, as well as providing high patient satisfaction and causing relatively few complications.

It can be difficult for inexperienced clinicians to preferentially recommend surgical treatment to patients, and patients also may be more interested in receiving non-surgical treatment. However, it should be remembered that surgical treatment is an important treatment option for some patients.

\section{REFERENCES}

1. Mulliken JB, Glowacki J. Hemangiomas and vascular malformations in infants and children: a classification based on endothelial characteristics. Plast Reconstr Surg 1982;69: 412-22.

2. Enjolras O, Mulliken JB. Vascular tumors and vascular malformations (new issues). Adv Dermatol 1997;13:375-423.

3. Neligan P. Plastic surgery. New York: Elsevier Saunders; 2013.

4. McCafferty I. Management of low-flow vascular malformations: clinical presentation, classification, patient selection, imaging and treatment. Cardiovasc Intervent Radiol 2015; 38:1082-104.

5. Lee BB. New approaches to the treatment of congenital vascular malformations (CVMs): a single centre experience. 
Eur J Vasc Endovasc Surg 2005;30:184-97.

6. Very M, Nagy M, Carr M, et al. Hemangiomas and vascular malformations: analysis of diagnostic accuracy. Laryngoscope 2002;112:612-5.

7. Roh YN, Do YS, Park KB, et al. The results of surgical treat- ment for patients with venous malformations. Ann Vasc Surg 2012;26:665-73.

8. Steiner F, FitzJohn T, Tan ST. Surgical treatment for venous malformation. J Plast Reconstr Aesthet Surg 2013;66:17419. 